\title{
Autoestima em praticantes de esportes de aventura
}

\author{
Self-esteem in practitioners of adventure sports
}

\author{
Rosane Camila Godoi, Hélio Mamoru Yoshida, Paula Teixeira Fernandes
}

Universidade Estadual de Campinas (Unicamp), Campinas/SP, Brasil

\section{HISTÓRICO DO ARTIGO}

Recebido: 18 novembro 2019

Revisado: 19 outubro 2020

Aprovado: 30 outubrio 2020

\section{PALAVRAS-CHAVE:}

Autoestima; Esportes de Aventura; Psicologia do Esporte; Aspectos Psicológicos; Natureza.

\section{KEYWORDS:}

Self-esteem; Adventure Sports; Sport Psychology; Psychology Aspects, Nature.

\section{RESUMO}

INTRODUÇÃO: Tendo em vista a atual ascensão dos esportes de aventura e dos diversos parâmetros psicológicos relacionados à essas práticas, faz-se necessário estudar como esses esportes, e seu risco inerente, são capazes de despertar distintas sensações e de que maneira isso pode influenciar a autoestima de seus praticantes. OBJETIVO: Esse estudo teve o objetivo de avaliar os índices de autoestima em praticantes de rafting, kayak, montanhismo e paraquedismo, avaliando os índices e analisando as correlações entre os grupos.

METODOS: Para isso, foram analisados os perfis de 73 praticantes de esportes de aventura (rafting, kayak, montanhismo e paraquedismo), utilizando-se como instrumento a Escala de Autoestima de Rosenberg, na versão adaptada por Hutz. O teste estatístico adotado foi o Kruskal-Wallis, a normalidade dos dados foi testada através do teste de Shapiro Wilk e todas as análises foram realizadas no programa Statistical Package for the Social Sciences.

RESULTADOS: Foi possível constatar elevados índices de autoestima em todos os participantes avaliados, em especial nos paraquedistas, sugerindo que os esportes de aventura são capazes de influenciar no bem-estar psicológico e a saúde mental de seus praticantes.

CONCLUSÃO: Com os dados obtidos, concluímos que este estudo traz o fortalecimento do bem-estar psicológico por meio da prática dos esportes de aventura, contribuindo para melhor autoestima e maior entendimento dos aspectos relacionados aos praticantes destas modalidades, ainda pouco estudados cientificamente.

\section{ABSTRACT}

BACKGROUND: In view of the current rise of adventure sports and the various psychological parameters related to these practices, it is necessary to study how these sports, and their inherent risk, are capable of awakening different sensations and how this could affect the self-esteem of its practitioners.

OBJECTIVE: This study aimed to evaluate the self-esteem indices in rafting, kayak, mountaineering and skydiving practitioners, evaluating the indexes, and analysing the correlations between the groups.

METHODS: For this, the profiles of 73 practitioners of adventure sports (rafting, kayak, mountaineering and skydiving) were analyzed using as an instrument the Rosenberg Self-Esteem Scale, in the version adapted by Hutz. The statistical test adopted was the Kruskal-Wallis, the normality of the data was tested using the Shapiro Wilk test, and all analyzes were performed using the Statistical Package for the Social Sciences program.

RESULTS: It was possible to verify the high self-esteem indices in all evaluated groups, especially skydivers, suggesting that adventure sports are capable of influencing the psychological well-being and mental health of their practitioners.

CONCLUSION: With these data, we can conclude that this study raises the question of psychological well-being through the practice of adventure sports, contributing to a better self-esteem and understanding of the aspects related to this portion of the population, which is still little studied. 


\section{INTRODUÇÃO}

Os esportes de aventura constituem um fenômeno social que tem se popularizado nos últimos anos. Parte do elemento motivador está relacionado à sua característica de instabilidade, mesmo que por vezes calculado e comedido, esse elemento de incerteza é inerente do próprio meio, o palco dessas práticas: a natureza (OLIVEIRA et al., 2018). Segundo Pereira, Armbrust e Ricardo (2008), a raiz dessa forma de expressão corporal mostra-se ligada à sociedade composta pela consciência significativa, sendo que esses tipos de atividade de risco são chamados de "afloramentos humanos" (PEREIRA; ARMBRUST; RICARDO, 2008, p.30).

Os esportes de aventura, por serem atividades praticadas ao ar livre envolvendo determinado risco, podem ser definidos como práticas na qual a habilidade de seu praticante, momento a momento, é fundamental para a manutenção de sua integridade física (BUCKLEY, 2018c). A partir disso, é possível observar distintas modalidades, como as praticadas em terra (montanhismo, escalada, rapel, mountain bike, exploração de caverna), em água (kayak, rafting, acqua ride, mergulho, canionismo), em meio aéreo (paraquedismo, vôo livre, balonismo), ou misto, quando duas ou mais modalidades interagem, como em corridas de aventura (PEREIRA; ARMBRUST; RICARDO, 2008).

Esse tema segue despertando interesse, uma vez que as atividades de aventura são capazes de proporcionar a fusão de distintas sensações, evidenciando a proximidade das práticas de aventura com as emoções de seus praticantes (LAVOURA; SCHWARTZ; MACHADO, 2008). O crescimento no número desses praticantes colabora para maior visibilidade para o segmento, que induz a diversificação das modalidades e a ressignificação dessa cultura corporal (ALVES; FONSECA; MARTINS, 2018).

De acordo com a literatura, esse tipo de atividade de aventura apresenta-se como um ambiente bastante rico e capaz de proporcionar contato mais profundo com diversos parâmetros psicológicos, entre as quais destacamos: diversas emoções (LAVOURA; SCHWARTZ; MACHADO, 2008); autoconceito (GIBBONS et al., 2018); promoção da saúde (BURR, 2013); autocontrole (GALOR; TENENBAUM; FURST, 1985). Nesse aspecto, a autoestima surge como uma variável interessante a ser estudada, uma vez que está fortemente ligada às percepções de habilidades pessoais, tão expostas nas atividades de aventura (BUCKLEY, 2018a).

Caracterizada como aspecto de autoconceito avaliativo, a autoestima é constituída por pensamentos, sentimentos e percepção de si mesmo frente ao mundo (HUTZ; ZANON, 2011). De maneira geral, o elevado índice de autoestima está associado a inúmeros benefícios, como bem-estar e eficiência em lidar com desafios, sendo uma resposta emocional ao avaliar e contemplar diferentes aspectos sobre si mesmo (HEATHERTON; WYLAND, 2003).

A autoestima também se mostra como uma importante variável para a boa saúde mental e o bem-estar psicológico, pois influencia na maneira como as pessoas enfrentam e solucionam os desafios com maior responsabilidade e confiança (NUNES et al., 2013).

Assim sendo, o objetivo desse estudo foi constatar e avaliar os índices de autoestima em praticantes de diferentes modali- dades de esportes de aventura, sendo escolhidos para esse estudo: kayak, rafting, montanhismo e paraquedismo, expondo, de maneira inédita, as principais correlações e distinções entre os praticantes dessas modalidades.

\section{MÉTODOS}

No total, 73 indivíduos participaram da pesquisa, nos quais 30 participantes eram do grupo kayak (41\%), 18 participantes eram da modalidade rafting (25\%), 14 do montanhismo (19\%) e 11 indivíduos do paraquedismo (15\%). A coleta foi feita no próprio ambiente de prática dos esportes de aventura. Foram convidados para a pesquisa praticantes dos seguintes esportes de aventura: kayak (especificamente a modalidade em águas brancas, ou seja, praticada em rios de corredeiras), rafting, montanhismo e paraquedismo, com idade de 18 a 55 anos de ambos os sexos, que por meio da assinatura do Termo de Consentimento Livre e Esclarecido (TCLE) deixaram explícito sua voluntariedade e concordância com a pesquisa. Como critérios de exclusão, foram desconsiderados aqueles que não se sentiram confortáveis em responder a pesquisa, a não concordância com a mesma, ou ainda o não preenchimento completo do instrumento utilizado.

O estudo foi conduzido em concordância com as recomendações e requisitos da Resolução no 466/2012 do Conselho Nacional de Saúde e suas Complementares, havendo compromisso na utilização de materiais e dados coletados exclusivamente para os fins previstos no protocolo e aprovado pelo Comitê de Ética em Pesquisa da Universidae de Campinas (CAAE no 69686017.0.0000.5404), sob o Parecer no 2.250.535.

As avaliações foram realizadas em locais especializados para a prática de rafting na cidade de Socorro (SP), no campeonato de kayak extremo Rio Selvagem, em Extrema (MG), travessias de montanha (SP e RJ) e no centro nacional de paraquedismo, em Boituva (SP). Todos esses locais e eventos contavam com participantes de todo o Brasil.

O instrumento escolhido foi a Escala de Autoestima de Rosenberg, adaptada por Hutz (HUTZ; ZANON, 2011). Este instrumento é bastante utilizado no meio científico e avalia a autoestima global por meio de dez afirmações relacionadas a sentimentos de autoestima e autoaceitação. Os itens são respondidos em escala do tipo likert de 4 pontos, com o escore final variando de 10 a 40 pontos, sendo que quanto maior o escore, maior o nível de autoestima.

Após aceito os termos da pesquisa e assinado o TCLE, os participantes preencheram a ficha de identificação e seguiram para a prática de costume e após concluí-la responderam a Escala de Autoestima. As condições de aplicação foram semelhantes para todos os participantes, sendo variável apenas o local e período de aplicação, que se estendeu de fevereiro a junho do ano de 2018.

Foi possível encontrar valores como média, desvio padrão, mediana, mínimo e máximo dos índices de autoestima. A estatística descritiva, através das medidas de posição e dispersão, foi utilizada para explorar o banco de dados coletados. O gráfico boxplot também foi utilizado para explorar e resumir os dados. A normalidade dos dados foi testada através do teste de Shapiro Wilk. O teste estatístico foi escolhido foi o Kruskal-Wallis para comparação entre as modalidades, tendo em vista a distribui- 
ção não normal dos dados para amostras não homogêneas. Todas as análises foram realizadas no programa Statistical Package for the Social Sciences - SPSS versão 23.0 para Windows. O nível de significância adotado foi de $p<0,05$.

\section{RESULTADOS}

Através da ficha de identificação pudemos observar que a maioria é do sexo masculino, constituindo $85 \%$ da amostra. A faixa etária dos indivíduos se estendeu de 18 a 55 anos, com idade média de $31 \pm 8,45$ anos, na seguinte proporção: 18 a 25 anos - 32,88\%, 26 a 35 anos - 39,74\%; 36 a 45 anos - 21,92\% e acima de 46 anos, 5,49\%.

Com relação ao tempo de prática, a média encontrada foi de $7,44 \pm 6,69$ anos, com as seguintes características: a maioria de praticantes com até 5 anos na modalidade $(53,42 \%)$, seguido da faixa entre 6 a 10 anos de prática $(20,55 \%)$ e com distribuição de 11 a 15 anos (12,33\%), no entanto, não raro, foi possível encontrar indivíduos que ultrapassam os 15 anos de prática nas 4 modalidades estudadas $(13,7 \%)$, chegando a registrar o máximo de 32 anos.

Além disso, os participantes responderam à seguinte pergunta: Você sente que houve mudança na sua vida (em qualquer aspecto) depois que começou a praticar esse esporte? Todos os participantes responderam, sendo que 98,63\% disseram SIM, e as justificativas - todas positivas - foram: melhora da saúde $(12,4 \%)$; qualidade de vida $(8,8 \%)$; autoconfiança/confiança $(8,8 \%)$; amizade $(6,6 \%)$; melhora física $(5,5 \%)$; bem-estar $(5,5 \%)$; concentração $(5,5 \%)$; diminuição do estresse $(4,4 \%)$; disciplina $(4,4 \%)$; autoestima (3,3\%); entre outros. Em relação aos índices de autoestima, os dados estão detalhados na Tabela 1. Interessante observar que, em uma escala em que a pontuação pode variar de 10 a 40, as médias encontradas foram superiores a 30 , o que demonstra elevados índices de autoestima nesses praticantes.

Tabela 1. Índices de Autoestima dos participantes do estudo, de acordo com a modalidade.

\begin{tabular}{lccccc}
\hline Modalidade ( $\mathbf{n})$ & Média & DP $( \pm)$ & Mín. & Md. & Máx. \\
\hline Rafting (18) & 34,7 & 4,24 & 26 & 35,5 & 40 \\
Kayak (30) & 34,1 & 4,38 & 26 & 35 & 40 \\
Montanhismo (14) & 33,6 & 4,22 & 27 & 34 & 40 \\
Paraquedismo (11) & 37,1 & 3,62 & 28 & 38 & 40 \\
\hline
\end{tabular}

Legenda: n: número de sujeitos; DP: Desvio Padrão, Mín: valores mínimos; Md: Mediana; Máx: valores máximos.

As análises mostraram que houve diferença estatisticamente significativa entre os níveis de estado de autoestima de kayak e paraquedismo $(p=0,005)$ e montanhismo e paraquedismo $(p=0,035)$, em ambas as comparações os praticantes de paraquedismo possuem maior nível de autoestima, como demonstra a Figura 1.

Foi possível observar a predominância do sexo masculino nas 4 modalidades estudadas. Composto apenas por $15 \%$ do total de participantes, esse dado evidencia que as mulheres ainda estão conquistando espaço nesse segmento. Uma hipótese levantada deve-se ao fato de que as mulheres ainda se deparam com estigmas em esportes considerados inóspitos e selvagens, porque vai de encontro ao culto à feminilidade, que associa o corpo feminino ao tipo frágil e inapto para esforços, o que restringe a vida social e física das mulheres brasileiras desde o século XIX (MOURÃO, 2000). Ainda nesse aspecto, a autora Ludmila Mourão (2000) nos recorda que mesmo que as mulheres carreguem o histórico de cerceamento de seus corpos, esse processo de infiltração e emancipação esportiva, inclusive nos es portes de aventura, ocorre através de um processo de inserção deveras lento, porém necessário.

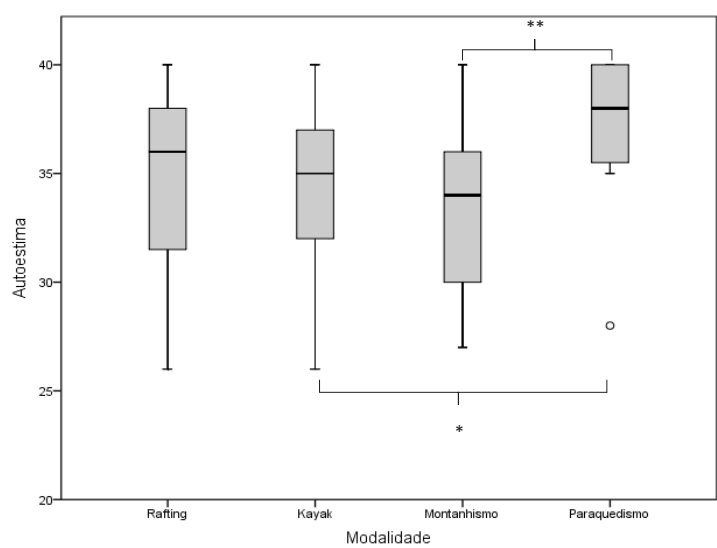

Figura 1. Média dos escores da Escala de Autoestima dos participantes do estudo, por modalidade.

Legenda: Valor dos escores do instrumento: 10 a 40 . Sendo 40 o valor máximo e 10 o valor mínimo. Rafting - Kayak: 0,159; Rafting - Montanhismo: 0,451; Rafting - Paraquedismo: 0,123, **Montanhismo - Paraquedismo: 0,035.

No que diz respeito ao tempo de prática, foi possível constatar que apesar da maioria dos grupos estar como jovens praticantes, com até 5 anos de prática $(53,42 \%)$, não foi difícil encontrar sujeitos que faziam do esporte de aventura seu veIho amigo, pois $13,7 \%$ praticavam sua modalidade esportiva de aventura há mais de 15 anos. Além disso, encontramos em nossos participantes um caso interessante: um dos indivíduos, com 44 anos de idade, é praticante de kayak há 32 anos. Isso pode nos sugerir que, após o início da prática, alguns indivíduos tendem a integrar o esporte de aventura como um "estilo de vida", que os acompanha ao longo da vida.

Referente à idade dos participantes, percebemos uma distribuição relativamente homogênea entre as diferentes faixas etárias, sendo composta por uma parcela menor $(5,49 \%)$ apenas na faixa que compreende as idades acima de 46 anos. Isso sugere que as atribulações da vida cotidiana, o interesse por outros assuntos, ou algum declínio físico e/ou psicológico tenha mais peso acima de determinada idade, demonstrando que antes disso, é possível que seu praticante possua esse estilo de vida que abrace seu esporte. Esse estilo de vida, proveniente dos esportes de aventura, é abordado por Buckley (2018b) como sendo uma trajetória de vida com ciclos de capacidades físicas e psicológicas de aventura recompensadoras, que perduram ao longo da vida (BUCKLEY, 2018b).

Esse mesmo autor faz considerações importantes no que se refere à aventura e ao envelhecimento, abrindo mais um diálogo interessante no qual podemos abordar o tempo de prática acompanhado do envelhecimento, processo natural e irremediável da condição humana (BUCKLEY, 2018a). Claramente os 
objetivos passam por transformação no decorrer dos anos, porém, a maior automotivação em praticar esportes, de maneira geral, surge mais frequentemente em grupos de pessoas de 24 a 54 anos (MORENO MURCIA et al., 2007), não sendo portanto, a idade um fator impeditivo direto para a realização da prática esportiva.

Buckley (2018a), cujo intuito era de estudar como o enveIhecimento poderia afetar a autoestima do praticante de esporte de aventura, afirma que envelhecer praticando esse tipo de esporte tem relação direta com a autoestima. De acordo com esse autor, a autoestima é capaz de refletir as habilidades atuais, olhar para as realizações passadas e para as aspirações futuras. Uma vez que ao envelhecer os esportistas tendem a reconhecer que estão se tornando mais lentos, fracos, mais facilmente cansados, mais suscetíveis, com mais lesões acumuladas e com resistência menor do que quando mais jovens, a autoestima aparece como fator psicológico naturalmente afetado durante este processo de envelhecimento. Porém, essa categoria de esportistas aprende a deixar uma margem de segurança e a construir estratégias à medida que suas percepções pessoais se modificam, pois percebem que suas capacidades estão sofrendo declínio, reduzindo então suas aspirações (BUCKLEY, 2018a).

Isso demonstra que os esportes de aventura, independentemente de quais, são capazes de abranger todos os tipos de pessoas e de promover a adaptação das habilidades ao esporte, compatíveis com a experiência na modalidade e a capacidade física de cada um. Vale enfatizar aqui que uma mesma modalidade pode ter percepções diferentes de desafio, fazendo com que o praticante seja privilegiado pela ampla gama de níveis de dificuldade, pois a vastidão da natureza adequa-se de acordo com o objetivo e grau de autonomia de cada pessoa. Um exemplo desse tipo de adequação de desafio seria uma pessoa ser capaz de descer grandes corredeiras em um rio, ou optar por trechos mais tranquilos; poder escolher entre o salto individual de paraquedas, ou a formação de figuras no ar (modalidade FQL: Formação em Queda Livre); poder escolher entre qualquer montanha do Brasil (baixa altitude), ou o Everest.

Para um atleta de aventura, a coragem é um importante componente da autoestima e, apesar de suas realizações e feitos comportarem-se como uma curva, aumentando até um pico em determinada idade (o que é bastante pessoal e varia por indivíduo), a descendência dessa curva não afeta negativamente a autoestima, ao contrário, sugere que a prática de esportes de aventura em geral tem efeito positivo (BUCKLEY, 2018a).

Esses desafios presentes nesse tipo de atividade fornecem poderoso impulso à autoestima, não disponível para não praticantes dessas modalidades. Uma possível explicação é o fato desses praticantes serem capazes de fixar novos objetivos de acordo com suas capacidades atuais, o que isola as perdas de autoestima. Uma outra explicação, em comparação com um estudo de Buckley (2018a), é de que um atleta de aventura em idade mais avançada, que teve toda uma vida de realizações não costuma olhar para trás para ganhar autoestima do passado, geralmente esse atleta olha para suas realizações passadas e sente-se satisfeito consigo mesmo e, então, traça aspirações futuras, comparando passado e futuro de um modo realista, mesmo que isso signifique ter objetivos e metas menos atléticas e audaciosas (BUCKLEY, 2018a).

Em referência ao instrumento utilizado nesse estudo, cujo escore de autoestima pode variar de 10 (mínimo) a 40 (máximo) pontos, observamos que as médias se mantiveram muito próximas nas 4 modalidades (kayak, rafting, montanhismo e paraquedismo), sendo um pouco mais elevada na modalidade paraquedismo em que a média se aproximou do valor máximo $(37,1)$, a mediana também se manteve próxima às médias, demonstrando que houve pouca variação dentro das modalidades, o que indica uma certa "homogeneidade de grupo". De fato, até mesmo as mínimas encontradas, que variaram de 26 a 28 , demostram uma autoestima considerada boa (escore acima de 25). Portanto, induz a premissa que os esportes de aventura colaboram para uma autoestima saudável, necessitando mais investigações para comprovar esse fenômeno.

Ainda sobre esse assunto, a elevada autoestima encontrada nas modalidades estudadas pode estar correlacionada com o fato de que uma elevada autoestima é um dos fatores de promoção na persistência (STENSENG; DALSKAU, 2010), posto que o tempo mínimo de prática foi de um ano.

Nesse possível paradoxo de se perguntar se quem tem elevada autoestima pratica mais esse tipo de esporte, ou se quem pratica esse esporte alimenta elevada autoestima, podemos destacar as respostas dadas sobre as mudanças que os praticantes notaram após o início do seu esporte: $98,63 \%$ disseram que sentiram mudança positiva em sua vida, presumindo que, ao praticar o esporte de aventura, o indivíduo inicia um círculo virtuoso, no qual a sua prática alimenta positivamente não somente a autoestima, mas, também o bem-estar físico e psicológico.

Outra condição que nos chama atenção é o elevado índice de autoestima presente nos paraquedistas, que destoa um pouco dos índices dos demais esportes. Uma possível hipótese seria que esse esporte, por ser considerado um dos mais extremos da categoria aventura, envolve outros parâmetros psicológicos que não foram abordados nesse estudo, talvez relacionados à autoeficácia, visto que é um aspecto provavelmente presente em indivíduos que saltam de um avião em movimento, talvez ligado à descarga de adrenalina, tão intensa nesse esporte. Sonstroem e Morgan (1988) mostraram que a percepção de autoeficácia pode contribuir para o aumento da autoestima e, dando início ao processo de retroalimentação, no qual o aumento da autoestima conduz à melhora na autoeficácia que, por sua vez, propicia maior percepção da autoestima global no praticante (SONSTROEM; MORGAN, 1988).

No entanto, é possível observar em outros estudos, aqui a exemplo de Betiollo e Santos (2002), que o montanhismo por si só também é capaz de desenvolver melhora na autoestima, pois ao apresentar situações de risco e incerteza pode incentivar perseverança, superação e confiança em si mesmo, promovendo inclusive posturas éticas e de educação ambiental (BETIOLLO; SANTOS, 2002).

Essas constatações corroboram com os dados encontrados, já que foi possível verificar os elevados índices de autoestima nos praticantes das 4 modalidades estudadas, independentemente do sexo, faixa etária ou tempo de prática. Dito isso, podemos relacionar a autoestima encontrada no presente estudo com um fator interessante a ser discutido: a possibilidade da relação direta com o desempenho na tarefa, uma vez que os sujeitos haviam acabado de praticá-la.

Nesse sentido, abordamos um outro estudo que também se utilizou da Escala de Autoestima de Rosenberg e desenvol- 
veu algumas percepções a partir desse olhar. De acordo com Stenseng e Dalskau (2010), a autoestima relaciona-se também ao engajamento em determinadas tarefas, podendo resultar de uma atividade passional. No caso, essa atividade passional é alusiva às práticas de aventura, e pode expor indivíduos obsessivamente engajados, que é descrito como um envolvimento controlado, conflituoso e inflexível em suas atividades; ou retratar uma paixão harmoniosa, relativo à um envolvimento equilibrado, autônomo e dinâmico em sua atividade (STENSENG; DALSKAU, 2010).

Essas avaliações comparativas de desempenho relacionado apenas à tarefa estão fortemente relacionadas ao nível mais baixo de autoestima, se comparado aos indivíduos harmoniosamente apaixonados por sua atividade (STENSENG; DALSKAU, 2010). Indivíduos com autoestima elevada e estável geralmente experimentam resultados positivos e negativos diariamente, mas, isso não afeta seus sentimentos globais de valor, pois são mais autodeterminados e tem autoconceito mais alto, pois, gostam, valorizam e aceitam a si mesmos (KERNIS, 2005).

Entretanto, os dados encontrados nesse estudo propõem que essa elevada autoestima se relaciona muito mais com a prática em si, do que com o desempenho, uma vez que nem todos tiveram o desempenho esperado, tal como os praticantes de kayak, que estavam em competição, mas, apenas 6 dos 30 atletas que responderam à pesquisa ganharam algum prêmio. No mais, as demais modalidades esportivas não eram competitivas, revelando que se a autoestima sofreu alguma influência direta, está mais relacionado simplesmente à prática em si, não ao seu desempenho durante a atividade.

Em um estudo recente, Woodman, MacGrego e Hardy (2020) investigaram a relação entre os benefícios de regulação de emoções aos benefícios associados a autoestima, e constataram que a participação em esportes de alto risco se associa também à autoestima elevada (WOODMAN; MacGREGOR; HARDY, 2020).

Para alguns estudiosos, a autoestima e a identidade própria estão diretamente ligadas às habilidades pessoais, avaliadas por si mesmo e pelos pares, utilizando-se de suas percepções e memórias (BUCKLEY, 2018a). A autoestima é um dos indicadores mais importantes para a boa saúde mental e para o bem-estar psicológico (NUNES, 2013; BUCKLEY, 2018), promovendo saúde e qualidade de vida (NUNES, 2013).

Logo, os aspectos psicológicos associados à essas práticas de aventura surgem como temas importantes a serem estudados, passíveis de infinitas possibilidades, lembrando que o contato do ser humano com a natureza promove ação educativa, com valores cooperativos e maior autonomia (ALVES; FONSECA; MARTINS, 2018).

Para além desses fatores, a troca de experiências e o enfrentamento coletivo do medo contribui para a inclusão em grupos, melhorando a autoestima e a promoção da saúde mental através da socialização (NUNES et al., 2013). Betiollo e Santos (2002) também observaram que a integração e a socialização estão bastante presentes nesses esportes, justamente por ser um ambiente rico em aprendizagens e trocas de experiências (BETIOLLO; SANTOS, 2002).

Por isso, partilhando da ideia de Piovani (2013), entende-se que o risco inerente dos esportes de aventura atua como elemento educador do ser humano, possibilitando a construção de pessoas autônomas, propiciando a tomada de decisões e a interação com o outro, favorecendo diferentes pontos de vista e emancipação, atingindo valores, conviç̧ões e crenças (PIOVANI, 2013).

Dessa maneira, deve-se levar em consideração os benefícios advindos dessas práticas, mesmo com possíveis riscos inerentes (BURR, 2013). A natureza é um ambiente subestimado cientificamente, pois o exercício ao ar livre é capaz de reduzir possíveis problemas de saúde, físicos e psicológicos (BUCKLEY, 2018a). Não obstante, precisamos nos ver como parte integrante e ativa da natureza, no qual o risco, intrínseco à mesma, é recurso psicológico capaz de nos transformar e nos fortalecer emocionalmente. Dessa maneira, podemos dizer que esse estilo de esporte se apresenta hábil para nutrir o círculo virtuoso capaz de desenvolver um conjunto de fatores necessários para uma boa saúde mental.

\section{CONCLUSÃO}

Com este estudo, pudemos observar que os praticantes de esportes de aventura, de maneira geral, apresentam elevada autoestima, estando relacionada ao bem-estar promovido tanto pela prática do esporte como pela interação do ser-humano com a natureza.

Como sugestão, deixa-se aberto a possibilidade da aferição da autoestima em um grupo controle para que seja plausível verificar possíveis diferenças com praticantes de esportes convencionais ou mesmo, pessoas sedentárias. No mais, a aplicação do instrumento foi feita logo após a prática de aventura e seria conveniente se houvesse a aplicação desse mesmo questionário no momento anterior à prática, para que se possa comparar alguma diferença significativa na autoestima entre o antes e o depois.

Portanto, diante dessas importantes constatações abre-se um novo horizonte de possibilidades a partir da Psicologia do Esporte e da Educação Física, principalmente no que se refere aos esportes de aventura em meio a natureza, panorama tão rico e ainda pouco explorado que irá contribuir para um melhor entendimento do comportamento humano.

\section{REFERÊNCIAS}

ALVES, M. P.; FONSECA, M. da C. V.; MARTINS, C. Educação física escolar, a prática de esportes de aventura e a noção de risco calculado. Linguagens, Educação e Sociedade, Teresina, v. 23, n. 38, p. 383-425, 2018.

BUCKLEY, R. C. Aging adventure athletes assess achievements and alter aspirations to maintain self-esteem. Frontiers in Psychology, Lausanne, v. 9, Article 225, p. 1-12, 2018.

BUCKLEY, R. C. Analysing adventure: a leisure lifepsychle? Annals of Leisure Research, Sydney, v. 21, n. 5, p. 533-8, 2018.

BUCKLEY, R. C. To analyze thrill, define extreme sports. Frontiers in Psychology, Lausanne, v. 9, Article 1216, p. 1-11, 2018.

BURR, J. F.; MONTELPARE, W. J.; SHEPHARD, R. J. Do adventure sports have a role in health promotion? Need for objective evidence for a risk-benefit analysis. Canadian Family Physician, Mississauga, v. 59, p. 1311-13, 2013.

GALOR, Y.; TENENBAUM, G.; FURST, D. Effect of self-control and anxiety on training performance in young and novice parachuters. Perceptual and Motor Skills, Netanya, v. 60, p. 743-6, 1985.

GIBBONS, S.; EBBECK, V.; GRUNO, J.; BATTEY, G. Impact of adventure-based approaches on the self- conceptions of middle school physical education students. Journal of Experiential Education, St. Petersburg, v. 41, n. 2, p. 220-32, 2018 
GUSTAFSSON, H.; MARTINENT, G.; GAUTHEUR, S. I.; HASSMÉN, P.; DESCAS, E. G. Performance based self-esteem and athlete-identity in athlete burnout: a person-centered approach. Psychology of Sport \& Exercise, Amsterdam, v. 38, p. 56-60, 2018.

HUTZ, C. S.; ZANON, C. Revisão da adaptação, validação e normatização da escala de autoestima de Rosenberg. Avaliação Psicológica, Porto Alegre, v. 10, n. 1. p. 41-9, 2011

LAVOURA, T. N.; SCHWARTZ, G. M.; MACHADO, A. A. Aspectos emocionais da prática de atividades de aventura na natureza: a (re)educação dos sentidos. Revista Brasileira de Educação Física e Esporte, São Paulo, v. 22, n. 2, p. 119-27, 2008.

MORENO MURCIA, J. A.; LÓPEZ DE SAN ROMÁN BLANCO, M.; MARTÍNEZ GALINDO, C.; VILLODRE, N. A.; GONZÁLEZ-CUTRE COLL, D. Efeitos do gênero, a idade e a frequência de prática na motivação e o desfrute do exercício físico. Fitness \& Performance Journal, Rio de Janeiro, v. 6, n. 3, p. 140-6, 2007.

MOURÃO, L. Representação social da mulher brasileira nas atividades físico-desportivas: da segregação à democratização. Movimento, Porto Alegre, v. 12 , n. 13, p. 5-18, 2000.

NUNES, M. R. M.; MONTIBELLER, C.; OLIVEIRA, K.; ARRACABA, R. C. B.; THEISS, S. M. M. B. Autoestima e saúde mental: Relato de experiência de um projeto de extensão. Psicologia Argumento, Curitiba, v. 31, n. 73, p. 2839, 2013.

PEREIRA, D. W.; ARMBRUST, I.; RICARDO, D. P. Esportes radicais, de aventura e ação: conceitos, classificaç̃̃es e características. Corpoconsciência, Santo André, v. 12, n. 1, p. 18-34, 2008.

PIOVANI, V. G. S. Atividades de aventura: prática para um tempo livre para o consumo ou para um tempo livre mais humano? Caderno de Educação Física e Esporte, Marechal Cândido Rondon, v. 11, n. 2, p. 61-7, 2013.

SONSTOEM, R. J.; MORGAN, W. P. Exercise and self-esteem: rationale and model. Medicine and Science in Sports and Exercise, Indianapolis, v. 21, n. 3, p. 329-37, 1989.

STENSENG, F.; DALSKAU, L. H. Passion, self-esteem, and the role of comparative performance evaluation. Journal of Sport \& Exercise Psychology, Champaign, v. 32, p. 881-94, 2010.

WOODMAN, T.; MacGREGOR, A. L.; HARDY, L. Risk can be good for self-esteem: beyond self-determination theory. Jornal of Risk Research, London, $v$. 23, n. 4, p. 411-23, 2020.

\section{AGRADECIMENTOS}

Os autores agradecem ao Grupo de Estudo em Psicologia do Esporte e Neurociência (GEPEN), da UNICAMP, por fomentar tantas discussões enriquecedoras e proporcionar a interação de pesquisadores de diversas áreas, despertando um diálogo impulsionador da ciência no Brasil

\section{CONFLITO DE INTERESSE}

Os autores do estudo declaram não haver conflito de interesses.

\section{FINANCIAMENTO}

Este estudo não teve apoio financeiro.

\section{ORCID E E-MAIL DOS AUTORES}

Rosane Camila Godoi (Autora Correspondente) ORCID: 0000-0003-1229-992X.

E-mail: r.camilagodoi@gmail.com

Hélio Mamoru Yoshida

ORCID: 0000-0002-1189-9192.

E-mail: heliomyoshida@gmail.com

Paula Teixeira Fernandes

ORCID: 0000-0002-0492-1670.

E-mail:paula@fef.unicamp.br 